\title{
Prevalence and mode of inheritance of major genetic eye diseases in China
}

\author{
DAN-NING HU \\ From the Zhabei Eye Institute, Shanghai, and Section of Ophthalmic Genetics, Chinese Society of Genetics
}

SUMMARY The prevalence and mode of inheritance of major genetic eye diseases have been investigated in China since the establishment of the Section of Ophthalmic Genetics of the Chinese Society of Genetics. Mass screening of genetic eye diseases has been undertaken in many districts in China, covering more than 700000 people, and more than 5000 pedigrees of genetic eye diseases have been collected and analysed all over China. Based on these data, the prevalence and mode of inheritance of dyschromatopsia, degenerative myopia, retinitis pigmentosa, congenital ptosis, congenital microphthalmos, congenital cataract, congenitalglaucoma, Leber's optic atrophy, corneal dystrophy, congenital nystagmus, coloboma of the eye $\bigcirc$ congenital aniridia, retinoblastoma, macular dystrophy, simple myopia, primary glaucoma, an strabismus have been investigated, and the results are presented.

The Section of Ophthalmic Genetics of the Chinese Society of Genetics was established in 1979. Before 1979, few ophthalmologists paid attention to the field of ophthalmic genetics, but since then an increasing number of ophthalmic genetic clinics and laboratories have been established in China. In June 1980, the First Chinese Congress of Ophthalmic Genetics was held. During this meeting, the prospects were also discussed for the Chinese Ophthalmic Genetics Programme Planning, the main content being to investigate the prevalence and mode of inheritance of major genetic eye diseases in China. The criteria for diagnosis and method of mass screening of genetic eye diseases were established and unified.

The Second, Third, and Fourth Chinese Congresses of Ophthalmic Genetics were held in 1982, 1984 , and 1986, respectively. Nine hundred and fifty-six ophthalmologists and geneticists attended these meetings, and 886 papers were read. Most of the Chinese ophthalmic centres joined our activities, including more than 500 members. Mass screening of genetic eye diseases has now been undertaken in many districts in China, covering 700000 people, and the results are presented in table 1 . More than 5000 pedigrees of genetic eye diseases have been collected and analysed from all over China. Based on these data, the prevalence and mode of inheritance of major genetic eye diseases have been documented.

Received for publication 23 December 1986

Accepted for publication 7 January 1987.
TABLE 1 Prevalence of major genetic eye diseases China.

\begin{tabular}{lll}
\hline Disease & $\begin{array}{l}\text { No of } \\
\text { people } \\
\text { examined }\end{array}$ & $\begin{array}{l}\text { Prevalence } \\
(\%)\end{array}$ \\
\hline Dyschromatopsia (male) & 278001 & $4 \cdot 89(1: 20)$ \\
Degenerative myopia & 26084 & $0 \cdot 95(1: 105)$ \\
Congenital ptosis & 247389 & $0 \cdot 18(1: 552)$ \\
Congenital cataract & 207319 & $0 \cdot 037(1: 2728)$ \\
Retinitis pigmentosa & 196777 & $0 \cdot 03(1: 3784)$ \\
Congenital nystagmus & 272931 & $0 \cdot 025(1: 4014)$ \\
Congenital microphthalmos & 310162 & $0 \cdot 009(1: 11077)$ \\
Congenital coloboma & 199525 & $0 \cdot 007(1: 15348)$ \\
Congenital glaucoma & 199525 & $0 \cdot 004(1: 24941)$ \\
Congenital aniridia & 267863 & $0 \cdot 00075(1: 133931)$ \\
\hline
\end{tabular}

\section{Congenital dyschromatopsia}

The prevalence of congenital dyschromatopsia (deutan and protan types) in 29 races in China have been thoroughly investigated. In the Han raceN which is the major racial group in China, the prevalence of dyschromatopsia in males was $4.89 \%$ (among $128357 \mathrm{men}$ ) and $\mathbf{0 . 7 \%}$ in females (among 56580 women). In some minor races, the prevalence् was lower or higher, for example, the prevalences? was only $1.75 \%$ in the Miao race (128 out of $5458_{0}$ men), but as high as $7.61 \%$ in the Hasaka race (D্D minor race of Caucasian origin). The mode of inheri tance of dyschromatopsia (deutan and protan types) i $\mathrm{X}$ linked recessive in China as in other countries. 


\section{Degenerative myopia}

The prevalence of degenerative myopia in East, Mid-south and North-east China was $0.95 \%$ (based on mass screening of 26084 people). $\mathrm{Hu}^{1}$ collected 61 pedigrees of degenerative myopia and found that it was inherited as an autosomal recessive trait; numerous other reports have been published since then (Geng, 87 pedigrees; Li, 96 pedigrees; Xia, 177 pedigrees; Nie, 16 pedigrees; Zhang, 70 pedigrees). All of these authors agreed that degenerative myopia was inherited as an autosomal recessive trait. When both parents were affected, 42 out of 45 offspring were affected $(93 \cdot 3 \%)$. When both parents of the probands appeared normal, $22.3 \%$ of the offspring were affected (Lenz's correction). When one of the parents of the probands was affected, 257 out of 564 offspring were affected $(45 \cdot 6 \%)$. These figures suggest autosomal recessive inheritance.

\section{Retinitis pigmentosa}

The prevalence of retinitis pigmentosa was $0.03 \%$ (1:3784) in East, Mid-south, North-west, Southwest, and North China (based on mass screening of 196777 people). Several large series of pedigrees of retinitis pigmentosa have been reported (320 pedigrees); the percentages of autosomal recessive (including sporadic cases), autosomal dominant, and $X$ linked recessive were $91.8 \%, 5 \cdot 2 \%$, and $3.0 \%$ respectively. Compared with data from Western countries, the percentages of $X$ linked recessive and autosomal dominant inherited retinitis pigmentosa in China is rather low, and the percentage of autosomal recessive retinitis pigmentosa is relatively high. ${ }^{2}$

\section{Congenital ptosis}

The prevalence of congenital ptosis in China was $0 \cdot 18 \%$ (1:552), based on mass screening of 247389 people. Two large series of pedigrees have been reported (76 pedigrees). The percentages of sporadic, autosomal dominant, and autosomal recessive cases were $67 \cdot 1 \%, 18.4 \%$, and $14.5 \%$ respectively. Ptosis with epicanthus inversus and blepharophimosis (Komoto's syndrome) is common in China, accounting for $8.3 \%$ of cases of congenital ptosis. It was inherited as an autosomal dominant trait with full penetrance.

\section{Congenital microphthalmos}

The prevalence of congenital microphthalmos was $0.009 \%(1: 11077)$ in 12 provinces in China, based on mass screening of 310162 people. In certain areas the prevalence was somewhat higher. For example, the prevalence was $0.057 \%(1: 1758)$ in East Henan. Most of the cases reported in China were sporadic ( 75 to $90 \%$ ); only 10 to $25 \%$ of cases had familial occurrence, all modes of inheritance being observed. Microphthalmos associated with aniridia or congenital cataract were inherited as autosomal dominant traits and most of the cases associated with corneal opacities or coloboma were sporadic or inherited as autosomal recessive traits.

\section{Congenital cataract}

The prevalence of congenital cataract was $0.037 \%$ (1:2728) in some districts in China, based on mass screening of 207319 people. Several large series of congenital cataract have been published. ${ }^{3}$ In 677 cases of congenital cataract reported in China, the percentages of sporadic, autosomal dominant, and autosomal recessive inheritance were $70.2 \%$, $21.0 \%$, and $8.8 \%$, respectively. Some $\mathrm{X}$ linked recessive cases have been reported also.

\section{Congenital glaucoma}

The prevalence of congenital glaucoma was $0.004 \%$ (1:24 941) in China based on mass screening of 199 525 people. A number of authors have reported large series of pedigrees of congenital glaucoma. In 234 cases reported in China, the frequency of parental consanguinity was $2 \cdot 3 \%$ (compared with an average of $1 \%$ in the general population). The corrected prevalence in sibships was 12.5 to $20.9 \%$, suggesting autosomal recessive inheritance. Since the prevalence was higher in males (male:female ratio $3 \cdot 3: 1$ ), and three cases of parent to child transmission have been reported, the possibility of multifactorial inheritance must also be considered.

\section{Leber's optic atrophy}

Many pedigrees of Leber's optic atrophy have been reported in China. ${ }^{4}$ The most striking feature is the relatively high incidence among females. In Chinese publications, we were able to find 354 cases, the ratio of affected females to males being $44.9 \%: 55 \cdot 1 \%$. This ratio is much higher than that reported in Western countries $(15 \%: 85 \%)$, but is similar to the Japanese figure $(41 \%: 59 \%) .^{5}$ The average age of onset was 18.8 years, which is earlier than that reported in Western countries and is close to the Japanese figure. ${ }^{5}$

\section{Corneal dystrophy}

The three most common types of corneal dystrophy 
reported in China were: nodular type (164 cases, inherited as autosomal dominant with high penetrance), reticular type (34 cases, inherited as autosomal dominant with high penetrance), and macular type (five pedigrees, inherited as autosomal recessive).

\section{Congenital primary nystagmus}

The prevalence of congenital nystagmus in China was $0.025 \%$ (1:4014, including both primary and secondary congenital nystagmus), based on mass screening of 272931 people. The Section of Ophthalmic Genetics of the Chinese Society of Genetics collected and analysed 208 pedigrees from 17 provinces. The mode of inheritance was sporadic in $64.4 \%$, autosomal dominant in $18.3 \%$, autosomal recessive in $8.7 \%, \mathrm{X}$ linked recessive in $7.2 \%$, and unknown in $1.4 \%$. No definite $X$ linked dominant inheritance has been identified.

\section{Congenital coloboma of the inner eye}

The prevalence of coloboma in Mid-south, Southwest, East, North-west, and North China was $0.007 \%(1: 15348)$, based on mass screening of 199525 people. The Section of Ophthalmic Genetics of the Chinese Society of Genetics collected and analysed 142 pedigrees from 21 provinces. Among 104 pedigrees of simple coloboma of the uvea, the percentages of sporadic, autosomal dominant, and autosomal recessive inheritance were $93.3 \%, 3.9 \%$, and $2.9 \%$ respectively. Among 12 pedigrees of coloboma of the macula, the percentages of sporadic, autosomal dominant, and autosomal recessive were $58 \cdot 3 \%, 33 \cdot 3 \%$, and $8 \cdot 3 \%$. Among 22 pedigrees of coloboma of the optic nerve, the percentages of sporadic and autosomal dominant cases were $86.4 \%$ and $13.6 \%$. Among four pedigrees of coloboma of the lens, two pedigrees showed sporadic and the other two autosomal dominant inheritance.

\section{Congenital aniridia}

The prevalence of aniridia in China was $0.00075 \%$ (1:133 931), based on mass screening of 267863 people. The mode of inheritance in 26 pedigrees reported in China was autosomal dominant with full penetrance.

\section{Retinoblastoma}

The incidence of retinoblastoma in living newborns in Shanghai was $1: 11800 .^{6}$ Most of the cases reported (1166 cases) were sporadic, only $2 \cdot 8 \%$ cases having a positive family history. ${ }^{7}$ The possible: explanation was that in the past the survival rate of retinoblastoma was low, so that most cases were either inherited through a carrier or occurred as new mutation. Bilateral cases accounted for $14.3 \% \frac{\text { }}{\widetilde{T}}$ Two cases of deletion of the long arm of chromo@ some 13 (13q14-) have been reported.

\section{Macular dystrophy}

In Stagardt's disease, the frequency of parenta consanguinity was $13.3 \%$ and the corrected pre valence among sibs was $23.8 \%$, indicating auto? somal recessive inheritance. In progressive cone-roф dystrophy, the frequency of parental consanguinity was $38.9 \%$ and the corrected prevalence among sibs was $28.2 \%$, indicating autosomal recessive inherio tance and heterogeneity.

\section{Simple myopia}

Simple myopia is common in China; in a mas screening of 130000 students in Liaoning Province in 1983 , the prevalence of reduced vision was $35.4 \%$ (myopia accounted for 85 to $95 \%$ of cases). In the large cities, the prevalence was high, being $59.4 \%$ in high school students in Shanghai city. There disagreement concerning the aetiology of myopias Some authors believe that myopia is related to genetic factors and others emphasise environment factors. Hu investigated this problem through a twi study. In 90 pairs of twins with myopia, th concordance rate of myopia was $81.6 \%$ in mono zygotic twins and $57.6 \%$ in dizygotic twins $(\mathrm{p}<0.05)$ The correlation coefficient was 0.71 in monozygot twins and 0.26 in dizygotic twins $(\mathrm{p}<0.01)$. The heritability index of myopia was $61 \% .^{8}$ Chu an Wang ${ }^{9}$ studied these problems in another groug of twins, with similar results: the heritability inde of myopia, radius of the cornea, axial length of the eyeball, and depth of the anterior chamber were $65 \%, 49 \%, 56 \%$, and $72 \%$, respectively. These results indicated that both genetic and environment factors play a role in the occurrence of myopia? Based on these data, Hu proposed a hypothesis of multifactorial inheritance of myopia. Li studied the role of inheritance of myopia in Shandong Province्ठ the familial occurrence rates in first and secono degree relatives of myopic patients were $15.1 \%$ and $6.3 \%$ and the prevalence of myopia in the same ares was $3 \cdot 3 \%$. The heritability of myopia as calculated from these data was $61.6 \%$. This pattern conformed with multifactorial inheritance.

\section{Primary glaucoma}

The prevalence of glaucoma in people over 30 yea 
TABLE 2 Recurrence risks of exotropia.

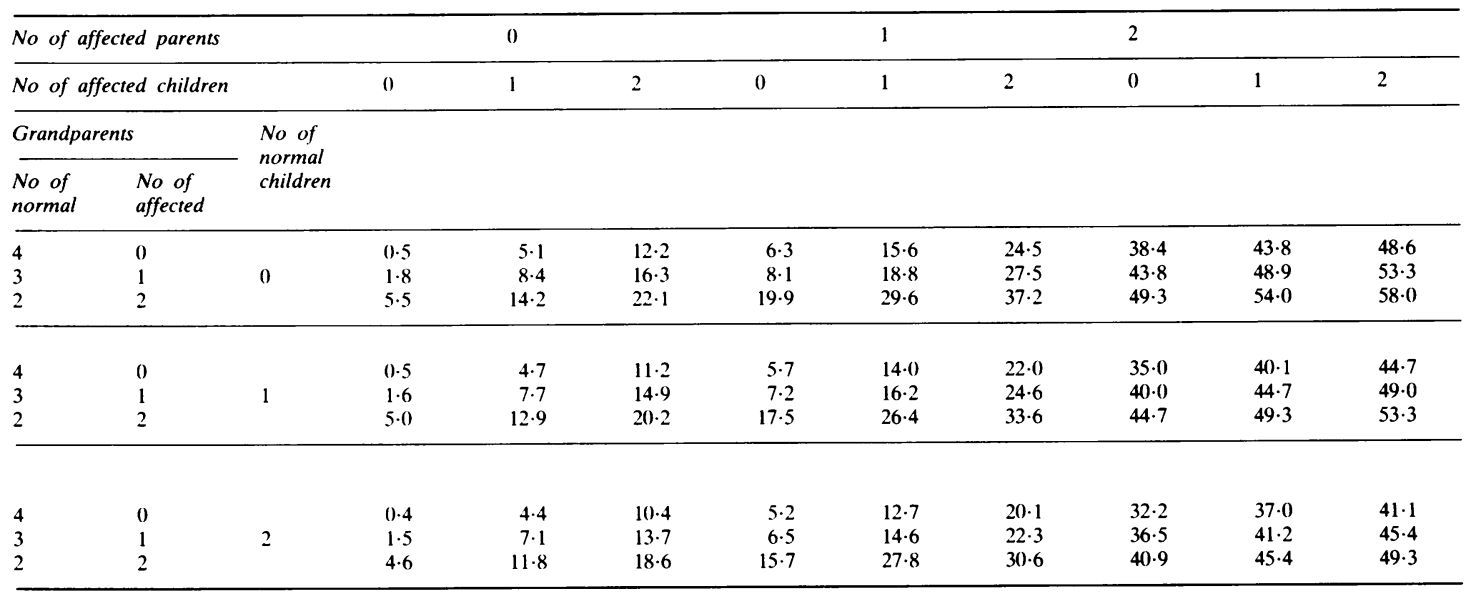

old was $1 \%$ in China. Closed angle glaucoma is more common than open angle glaucoma in China. Wang investigated the mode of inheritance of primary closed angle glaucoma in 100 cases. The familial occurrence rates in first and second degree relatives was $7 \cdot 1 \%$ and $2 \cdot 33 \%$, respectively. The prevalence of closed angle glaucoma in the same district was $0.96 \% .^{10}$ The heritability of closed angle glaucoma as calculated from these data was $65 \%$.

\section{Strabismus}

$\mathrm{Hu}$ investigated the mode of inheritance of exotropia in 425 persons. The familial occurrence rate in first, second, and third degree relatives was $9.0 \%$, $2 \cdot 2 \%$, and $1 \cdot 1 \%$ respectively. The prevalence of exotropia was $0.58 \%$. The heritability of exotropia as calculated from these data was $81 \cdot 3 \%$.

\section{Other genetic eye diseases}

\section{CRYSTALLINE RETINOPATHY}

This is a very rare disease in Western countries, according to Grizzard et al. ${ }^{11}$ There have been only 14 reported cases in the West, but it is quite common in China. During 1978 to 1980 , we observed 25 families ( 35 patients) in our clinic. All of the parents and offspring of the probands appeared normal, but some of their sibs were affected, the corrected prevalence among the sibs being $19 \cdot 2 \%$. The frequency of parental consanguinity was $20.0 \%$, indicating autosomal recessive inheritance. reported in Japan, but only 26 cases have been reported in Caucasians. There have been 16 cases reported in China. Families with several cases in sibs and consanguinity of parents have been reported, indicating autosomal recessive inheritance.

\section{Genetic counselling}

We established a clinic for genetic eye disease and genetic counselling in 1978 . More than 1500 patients have visited the clinic or have written for genetic counselling. We have designed genetic counselling tables for some monogenic inherited eye diseases, including degenerative myopia, corneal dystrophy, and aniridia, based on the genetic percentages, mode of inheritance, degree of penetrance, and the gene frequency among the Chinese.

We have developed a computer programme to estimate recurrence risks for multifactorial inherited genetic diseases based on Smith's formula. ${ }^{12}$ Using this programme, we have prepared tables for estimating recurrence risks for a variety of family situations in several multifactorial inherited eye diseases, such as exotropia and primary closed angle glaucoma (table 2).

\section{References}

1 Hu DN. Genetic aspects of high myopia. Chin J Ophthalmol 1979:15:159-62.

${ }^{2} \mathrm{Hu}$ DN. Genetic aspects of retinitis pigmentosa in China. Am J Med Genet 1982;12:51-6.

3 Jin YZ, Shun Z, Dou SH. Congenital cataract: investigation and analysis of eighty two pedigrees. Chin J Ophthalmol 1983;19: 296-7.

4 Ton Y. Analysis of fifty eight pedigrees of Leber's optic atrophy. Chin J Ophthalmol 1985;21:163-5. 
5 François J. Heredity in ophthalmology. St Louis: Mosby, 1961:402-4, 497-508.

- Wang GM, Guo BK, Chu RY. Retinoblastoma in Shanghai. Chin J Ophthalmol 1985;21:288-92.

7 Zhang PY, Ling DQ, Guo YS, Chang BX. Genetics of retinoblastoma. Chin J Ophthalmol 1983;19:201-3.

${ }^{8}$ Hu DN. Twin study on myopia. Chin Med J 1981;94:51-5.

9 Chu RY, Wang GM. Study of myopia occurring in twins. Chin J Ophthalmol 1983;19:266-8.

10 Wang RR, Guo BK, Ji XZ, Cheng SZ. Genetics of closed-angle glaucoma. Chin J Ophthalmol 1985;21:95-101.
"Grizzard WS, Deutman AF, Nijhuis F, DeKerk J. Crystall $\overrightarrow{\text { קe }}$ retinopathy. Am J Ophthalmol 1978;86:81-8.

12 Smith C. Recurrence risks for multifactorial inheritance. A $J$ Hum Genet 1971;23:578-84.

Correspondence and requests for reprints to Dr DôN Hu, Room 402, 490 Shan Xi Nan Road, Shanghgi, China. 